\title{
Periventricular nodular heterotopia in a child with a mild Mowat-Wilson phenotype caused by a novel missense mutation of ZEB2
}

\author{
Young Ok Kim ${ }^{1, *}$, Yun Young Lee ${ }^{2 \oplus}$, Myeong-Kyu Kim ${ }^{3 \oplus}$, and Young Jong Woo ${ }^{1 \oplus}$ \\ 'Department of Pediatrics, Chonnam National University Medical School, Gwangju, Korea \\ ${ }^{2}$ Department of Radiology, Chonnam National University Hospital, Gwangju, Korea \\ ${ }^{3}$ Department of Neurology, Chonnam National University Medical School, Gwangju, Korea
}

Periventricular nodular heterotopia $(\mathrm{PNH})$ is a malformation of cortical development in which normal neurons inappropriately cluster in periventricular areas. Patients with Mowat-Wilson syndrome (MWS) typically present with facial gestalt, complex neurologic problems (e.g., severe developmental delay with marked speech impairment and epilepsy), and multiple anomalies (e.g., Hirschsprung disease, urogenital anomalies, congenital heart defects, eye anomalies, and agenesis of the corpus callosum [CC]). MWS is mostly caused by haploinsufficiency of the gene encoding zinc-finger E-box-binding homeobox 2 (ZEB2) due to premature stops or large deletions. We present a case report of a 9-year-old girl with $\mathrm{PNH}$, drug-responsive epilepsy, severe intellectual disability, and facial dysmorphisms only in whom we performed whole-exome sequencing and found a de novo heterozygous missense mutation (c.3134A>C; p.His1045Pro) of ZEB2 (NM_014795.3; NP_055610.1). This mild case of MWS caused by a rare novel missense mutation of ZEB2 represents the first report of MWS with isolated PNH.

Key words: Periventricular nodular heterotopia, Zinc finger E-box binding homeobox 2, Seizures, Intellectual disability.

\section{Introduction}

Periventricular nodular heterotopia (PNH) is the most common neuronal heterotopia, and it can exist in an isolated form or can appear with other brain malformations [1]. Two genes have been reported previously in individuals with diffuse forms of PNH: that encoding filamin A (FLNA) and that encoding ADPribosylation-factor guanine nucleotide exchange factor 2 (ARFGEF2) [1]. Certain chromosomal abnormalities such as duplication in $5 p 15.1$ or $5 p 15.33$ and deletion in 5q14.3, 6p25, 6q27, or 7q11.23 have also been reported [1].

Mowat-Wilson syndrome (MWS) is a severe intellectual disability syndrome that manifests with typical facial dysmorphic features and malformations in multiple organs [2-12]. It is caused by mutations of the gene encoding zinc-finger E-boxbinding homeobox 2 (ZEB2) on chromosome 2q22.3, most of which are truncations, frame shifts, or large deletions, whereas missense mutations have been quite rare and have usually resulted in a mild phenotype $[4,9,10,12]$.

We used whole-exome sequencing (WES) in an attempt to

\footnotetext{
Received: 17 September 2019, Revised: 18 October 2019, Accepted: 1 November 2019, Published: 31 December 2019

*Corresponding author: Young Ok Kim, M.D., Ph.D. (iD https://orcid.org/0000-0002-7873-1140

Department of Pediatrics, Chonnam National University Childrens' Hospital, 42 Jebong-ro, Dong-gu, Gwangju 61469, Korea.

Tel: +82-62-220-6646, Fax: +82-62-222-6103, E-mail: ik052@jnu.ac.kr

Conflict of interest: The authors declare that they do not have any conflicts of interest.

(c) This is an open-access article distributed under the terms of the Creative Commons Attribution Non-Commercial License (http://creativecommons.org/licenses/by-nc/4.0/) which permits unrestricted non-commercial use, distribution, and reproduction in any medium, provided the original work is properly cited.

(c) Copyright 2019 by the Korean Society of Medical Genetics and Genomics 
identify the genetic etiology in a 9-year-old girl with isolated PNH, epilepsy, severe intellectual disability, and mild facial dysmorphism, which revealed a very rare missense mutation of ZEB2. The current case with a mild form of MWS had typical facial features in addition to compatible neurologic problems, but none of the well-known anomalies in other internal organs. $\mathrm{PNH}$ is very rare in MWS, and this might be the first report of isolated PNH without other brain malformation in MWS.

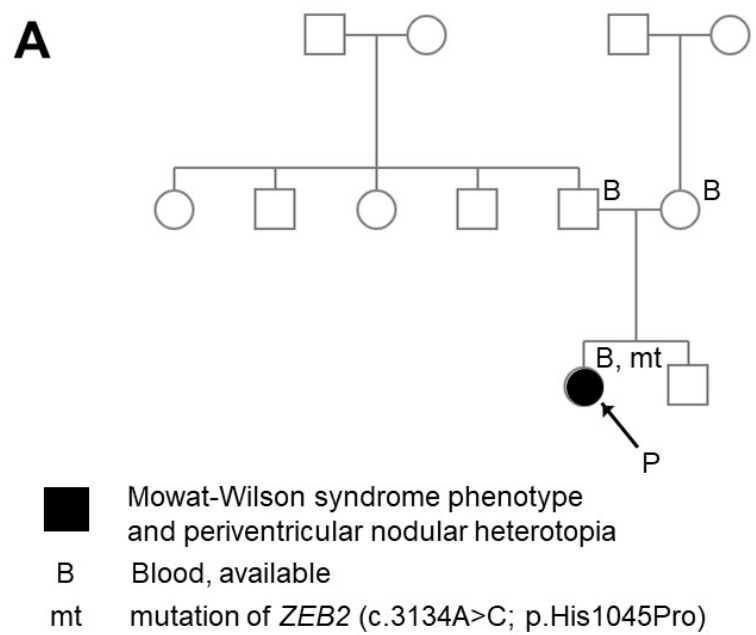

B
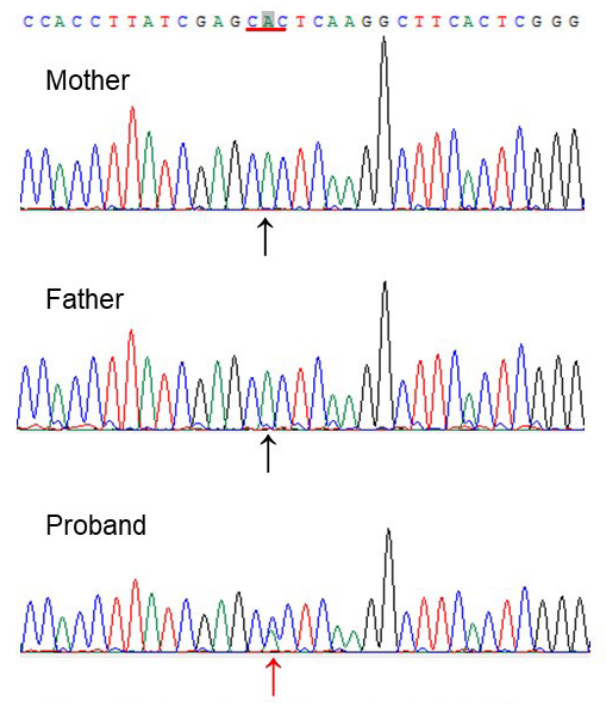

Novel likely-pathogenic variant of ZEB2 (c.3134A>C; p.His1045Pro)

Fig. 1. Family pedigree of the proband with Mowat-Wilson syndrome (A) and electropherogram of the novel heterozygous mutation of ZEB2 (B). Whole-exome sequencing performed in the proband found the likely pathogenic variant of $Z E B 2$ in the proband, with the sequences verified using Sanger sequencing in trio. The variant was novel in that it was found only in the proband (red arrow) and not in her parents (black arrow) (B).

\section{Case}

\section{Patients}

The female proband with severe intellectual disability visited our hospital at 9 years and 7 months of age due to bilateral tonic-clonic seizures without awareness that were associated with eyeball deviation, and lasted $3 \mathrm{~min}$. She was born at a gestational age of 40 weeks weighing $3.7 \mathrm{~kg}$ with a normal-sized head after an uneventful pregnancy. Her parents were healthy and unrelated. Her younger brother developed normally. There was no family history of seizures or developmental delay (Fig. 1)

She had experienced recurrent tonic-clonic seizures with eyeball deviation from 8 months of age, at which time she already exhibited global developmental delay. Her seizures were usually precipitated by fever, which occurred once or twice yearly, and she was not taking daily antiepileptic drug medication. Her electroencephalogram (EEG) had been checked yearly before school entrance, and all of the recordings were normal. She had facial dysmorphic features: frontal bossing, broad and horizontal eyebrows with medial flaring, hypertelorism, deep-set and large eyes with upward-slanted palpebral fissures, a broad and depressed nasal bridge, a round nasal tip, a prominent columella with a short philtrum, an M-shaped upper lip, a pointed and triangular chin, and large and posteriorly rotated ears with an uplifted fleshy ear lobe that was centrally depressed. Spine teleoroentgenography showed lumbar scoliosis. However, echocardiography and abdominal ultrasonography revealed no anomalies in the internal organs. Brain magnetic resonance imaging (MRI) on admission showed subependymal nodular heterotopia along the bilateral ventricles (Fig. 2). EEGs measured during sleep showed diffuse, rhythmic, and frontal-dominant slow delta-wave bursts with or without spikes (Fig. 3). Metabolic

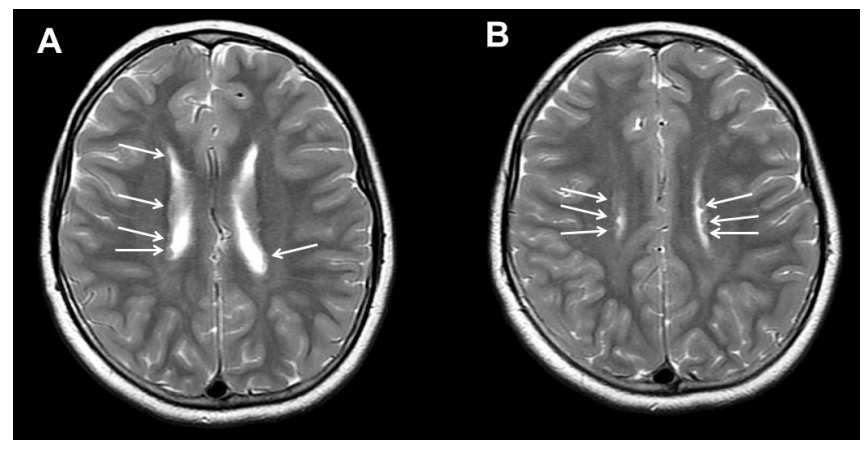

Fig. 2. Brain T2-weighted axial magnetic resonance imaging. (A, B) Small nodular lesions (arrows) isointense with gray matter appeared lining the lateral wall of both lateral ventricles, which indicates subependymal gray-matter heterotopia. 


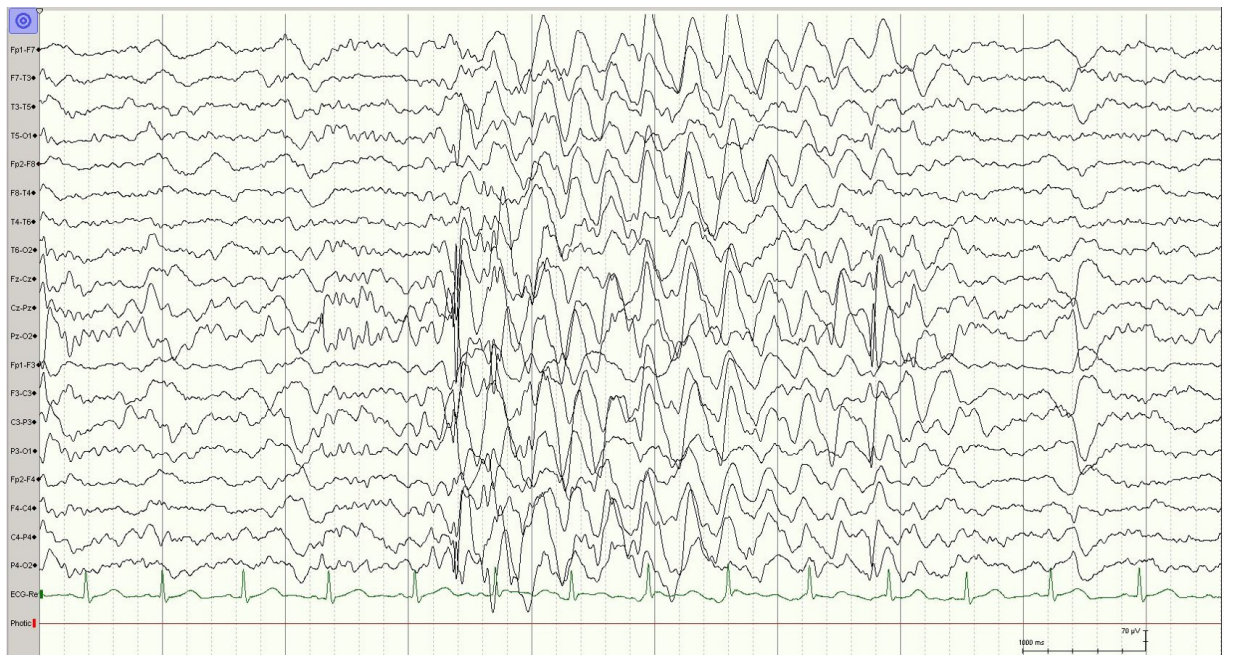

Fig. 3. Electroencephalogram (EEG) of the proband at 9 years and 7 months of age. EEGs measured during sleep showed diffuse, rhythmic, and frontal-dominant slow delta-wave bursts. screening during the neonatal period had produced normal findings, and her blood levels of lactate and ammonia, serum electrolytes, and blood amino-acid analysis on her current visit were all normal. A chromosomal analysis and chromosomal microarray study produced no positive results. Her seizures were easily controlled by initial topiramate and subsequent levetiracetam, and she experienced no further seizures during a 15-month follow-up.

\section{Molecular genetic analysis}

WES was performed in the proband using SureSelect Human All Exon probes (version 5; Agilent Technologies, Santa Clara, CA, USA) for library preparation on the Illumina NextSeq500 platform (Illumina Inc., San Diego, CA, USA). The alignment of sequence reads, indexing of the reference genome (hg 19), and variant calling with a pipeline were based on the GATK (Genome Analysis Tool Kit, version 3.5) Best Practice. According to our bioinformatics flow, the variants were prioritized while referring to different databases of single-nucleotide polymorphisms (SNPs), including the Korean Reference Genome Database (http://coda.nih.go.kr/coda/KRGDB/index.jsp). A novel heterozygous missense variant (c.3134A>C; p.His1045Pro) of ZEB2 (NM_014795.3; NP_055610.1) was found, which was not found in any SNP databases. The mutation site of the base was assumed to be highly conserved and was predicted as deleterious in silico. Sanger sequencing confirmed that the mutation was de novo, as it was not found in her parents (Fig. 1). This variant was classified as a likely pathogenic one according to the guidelines reached by a joint consensus of the American College of Medical Genetics and Genomics and the Association for Molecular Pathology [13].
This study was approved by the Human Research Ethics Committee of Chonnam National University Hospital (approval number: CNUH-2017-167). Informed consents were obtained from her parents. The biospecimens were provided by the Chonnam National University Hospital Biomedical Research Institute Biobank according to the protocols approved by the review board.

\section{Discussion}

PNH consisting of ectopic gray-matter nodules in single or multiple forms induces irregular protruding lines of the ventricle, whose nodules appear to be quite epileptogenic [1]. Typically, 80\% to 90\% patients with PNH are thought to have epilepsy, with the associated seizures mostly being focal and drug-responsive, although they can be drug-resistant in some patients [1]. Intellectual disability and developmental delay are other common problems in patients with $\mathrm{PNH}_{1}$ and are more severe in cases with additional brain malformations [1]. In the current case with isolated $\mathrm{PNH}$, we first considered the possibility of other genes or chromosomal loci known to be present in diffuse PNH without other brain malformation-it was not easy to guess the ZEB2 mutation due to her mild phenotypes of MWS without the well-known anomalies in other internal organs.

All MWS patients exhibit moderate-to-severe intellectual disability, and 98\% of patients with ZEB2 mutations show the following facial characteristics that change with age: a high and broad forehead with bossing; medially flaring, thick, but centrally sparse eyebrows; hypertelorism; large and deep-set eyes; strabismus, and downward-slanting palpebral fissures; uplifted large and fleshy ear lobes with a central depression 
such as 'orecchiette pasta'; a broad nasal bridge and rounded tip; a prominent columella making the philtrum appear short; an M-shaped upper lip with open mouth; a pointed chin with a triangular jaw $[7,8,11,12]$. Our patient presented with mild manifestations of some of the characteristic features of facial gestalt in MWS.

Patients with MWS also exhibit multiple congenital anomalies with variable occurrence rates: microcephaly (77.7\%); hypoplasia or agenesis of the CC (46\%); Hirschsprung disease (44.2\%); pyloric stenosis (7.4\%); congenital heart diseases (58.1\%); hypospadias (59.7\%); cryptorchidism (41.5\%); renal anomalies (25.3\%); short stature (46.4\%); structural eye anomalies (9.9\%); cleft palate (1.8\%); and pulmonary artery sling $(3.4 \%)[7,8,11,12]$. Anomalies of the central nervous system (CNS) are the most commonly reported $[7,8,11,12]$.

According to Garavelli and colleagues, who reviewed brain MRI findings for 54 MWS patients with ZEB2 mutations, the rate of CNS anomalies (44.6\%) appears to be underestimated in the literature, since they found that most of their patients $(n=52$, $96 \%$ ) had abnormal results $[2,7,8,11]$.These findings included anomalies of the CC (79.6\% versus $46 \%$ in the previous literature); hippocampal abnormalities (77.8\% versus $7.1 \%)$; ventriculomegaly (68.5\% versus 12.5\%); white-matter abnormalities (reduction of thickness, $40.7 \%$ versus $17.9 \%$; localized signal alterations, 22.2\% versus no report); cerebellar malformation and Chiari type 1 malformation (11.1\%); cortical malformation such as polymicrogyria, $\mathrm{PNH}$, and focal cortical dysplasia (7.4\%); large basal ganglia (5.6\%); and brain tumor (1.9\%) $[2,3,7,8,11]$. One patient with $\mathrm{PNH}$ who had whole-allele deletion of ZEB2 showed complete agenesis of the $\mathrm{CC}_{\text {, enlarged ventricles, and }}$ hippocampal abnormalities [2]. The isolated PNH in the present case might have been due to a mild phenotype associated with missense mutations of ZEB2.

Seizures are reported in $78.5 \%$ patients with MWS, and they usually appear around the second year of age and have diverse phenotypes $[2,5,7,8,11,12]$. According to Cordelli et al. [5], most cases initially present with focal seizures, with atypical absence appearing after 4 years in some cases. Seizures are often provoked by fever and frequently occur during sleep [5]. EEGs at onset are normal or show mild slowing, which later changes into frontal-dominant spike-and-wave discharges [5]. The effectiveness of antiepileptic drugs reportedly varies in individuals with MWS, with drug-resistant seizures becoming responsive after adolescence in some cases $[2,5,8,11]$. Our patient had drugresponsive seizures from 8 months of age that were provoked by fever: the EEG after 9 years showed frontal-dominant diffuse slow delta-wave bursts with or without spikes. Behavioral characteristics of MWS have included a happy and social demeanor with a smiling face, and body or oral stereotypes $[6,8]$.

Missense ZEB2 mutations have only very rarely been identified in patients with MWS, with most cases showing a mild or atypical phenotype $[4,9,10]$. Ghoumid et al. [4] reported in 2013 that there only six missense mutations had been described until then, including three of their own cases $[9,10]$. One of their patients (Patient 1) had a mutation (c.3134A>G; p.His1045Arg) at the same base site as in our case, and showed mild facial gestalt of MWS with a normal-sized head and moderate intellectual disability, and no cardiac malformation or Hirschsprung disease, but he had hippocampal anomalies and frontal cortical atrophy [4]. However, he had never experienced seizures.

The rare missense mutation of ZEB2 in the present 9-yearold girl resulted in isolated $\mathrm{PNH}$ in addition to drug-responsive epilepsy, severe intellectual disability, and mild facial gestalt of MWS. This is the first report of isolated PNH in MWS.

\section{Acknowledgements}

This research was supported by the Basic Science Research Program through the National Research Foundation of Korea (NRF) funded by the Ministry of Education, Republic of Korea (grant no. NRF-2017R1D1A3A03000532).

\section{References}

1. Guerrini R, Dobyns WB. Malformations of cortical development: clinical features and genetic causes. Lancet Neurol 2014;13:710-26.

2. Garavelli L, Ivanovski I, Caraffi SG, Santodirocco D, Pollazzon M, Cordelli DM, et al. Neuroimaging findings in Mowat-Wilson syndrome: a study of 54 patients. Genet Med 2017;19:691-700.

3. Murray SB, Spangler BB, Helm BM, Vergano SS. Polymicrogyria in a 10-month-old boy with Mowat-Wilson syndrome. Am J Med Genet A 2015:167A:2402-5.

4. Ghoumid J, Drevillon L, Alavi-Naini SM, Bondurand N, Rio M, BriandSuleau $A$, et al. ZEB2 zinc-finger missense mutations lead to hypomorphic alleles and a mild Mowat-Wilson syndrome. Hum Mol Genet 2013;22:2652-61.

5. Cordelli DM, Garavelli L, Savasta S, Guerra A, Pellicciari A, Giordano L, et al. Epilepsy in Mowat-Wilson syndrome: delineation of the electroclinical phenotype. Am J Med Genet A 2013;161A:273-84.

6. Evans E, Einfeld S, Mowat D, Taffe J, Tonge B, Wilson M. The behavioral phenotype of Mowat-Wilson syndrome. Am J Med Genet A 2012;158A:358-66. 
7. Garavelli L, Zollino M, Mainardi PC, Gurrieri F, Rivieri F, Soli $F$, et al. Mowat-Wilson syndrome: facial phenotype changing with age: study of 19 Italian patients and review of the literature. Am J Med Genet A 2009;149A:417-26.

8. Garavelli L, Mainardi PC. Mowat-Wilson syndrome. Orphanet J Rare Dis 2007;2:42.

9. Dastot-Le Moal F, Wilson M, Mowat D, Collot N, Niel F, Goossens M. ZFHX1B mutations in patients with Mowat-Wilson syndrome. Hum Mutat 2007;28:313-21.

10. Heinritz W, Zweier C, Froster UG, Strenge $S$, Kujat $A$, Syrbe $S$, et al. A missense mutation in the ZFHX1B gene associated with an atypical Mowat-Wilson syndrome phenotype. Am J Med Genet A
2006;140:1223-7.

11. Mowat DR, Wilson MJ, Goossens M. Mowat-Wilson syndrome. J Med Genet 2003;40:305-10.

12. Ivanovski I, Djuric O, Caraffi SG, Santodirocco D, Pollazzon M, Rosato S, et al. Phenotype and genotype of 87 patients with Mowat-Wilson syndrome and recommendations for care. Genet Med 2018;20:96575.

13. Richards S, Aziz N, Bale S, Bick D, Das S, Gastier-Foster J, et al. Standards and guidelines for the interpretation of sequence variants: a joint consensus recommendation of the American College of Medical Genetics and Genomics and the Association for Molecular Pathology Genet Med 2015;17:405-24. 